\title{
Relación entre el uso del chat y los juegos en red en las habilidades sociales de los alumnos de primer y segundo año de Ingeniera de Sistemas y Psicología de la Universidad Peruana Unión
}

\author{
Lindsey Wildman VILCA QUIRO', Gabriela GONZALES YUPANQUI² y Walter CAPA LUQUE ${ }^{3}$
}

\begin{abstract}
RESUMEN
Objetivo: Determinar la relación del uso del chat y los juegos en red en las habilidades sociales de los alumnos de primer y segundo año de Ingeniera Sistemas y Psicología. Metodología: Se empleó una muestra no probabilística de muestreo por conveniencia de estudiantes universitarios de la Universidad Peruana Unión de Lima, incluyendo universitarios de ambos sexos con edades entre 15 y 25 años. Resultados: Los resultados indican que los alumnos de la carrera de Ingeniería de Sistemas (50\%) se caracterizan por hacer un uso moderado del chat. Por otro lado, se observa que los de Psicología (50\%) a comparación de los de Ingeniería de Sistemas (16.3\%) utilizan muy poco el chat. En cuanto a los juegos en red, en Psicología (96.4\%) se caracterizan por jugar muy poco juegos en red al igual que la carrera de Ingeniería de Sistemas (63.8\%). Aunque los de la carrera de Ingeniería de Sistemas (30\%) presentan un nivel de uso promedio de los juegos en red y con respecto a habilidades sociales tenemos que en la carrera de Psicología (97.6\%) se caracterizan por tener un nivel de habilidades sociales altas. De manera similar también ocurre con la carrera de Ingeniería de Sistemas (91.3\%).Conclusiones: Por lo tanto, la relación de correlación nos indica que existe una relación negativa y altamente significativa entre el uso del chat y las habilidades sociales ( $\mathrm{r}$ $=-0.419, \mathrm{p}<0.01)$. Esto quiere decir que cuanto menor es el nivel de uso del chat, mayor es el nivel de habilidades sociales. Asimismo, existe una relación negativa y altamente significativa entre el uso de los juegos en red y las habilidades sociales $(r=-0.215, \mathrm{p}<0.01)$. Esto quiere decir que cuanto mayor es el uso de los juegos en red menor son las habilidades sociales.
\end{abstract}

Palabras clave: Habilidades sociales, chat, juegos en red, comunicación.

\begin{abstract}
Objective: To determinate the relate that exist between the use of chat and online games on social skills of students in first and second years of Systems Engineering and Psychology. Methods: We used a nonrandom sample of convenience sample of university students from the Universidad Peruana Unión of Lima, including college students of both sexes aged between 15 and 25. Results: The results indicate that students in the Engineering Systems $(50 \%)$ are generally characterized by moderate use of chat. On the other hand, we observe that psychology (50\%) compared to systems engineering (16.3\%) used very little chat. As for gaming, Psychology $(96.4 \%)$ is characterized by very little play online games like the Systems Engineering degree (63.8\%). Although the Engineering Systems (30\%) had a level of average usage of online gaming, and with respect to social skills have to Psychology course (97.6\%) were characterized by a high level of social skills. Similarly also applies to the Engineering Systems (91.3\%). Conclusions: Therefore the correlation ratio indicates that there is a highly significant negative relationship between the use of chat and social skills $(\mathrm{r}=-0.419, \mathrm{p}<0.01)$. This means that the lower the level of use of chat higher the level of social skills. There is also a highly significant negative relationship between the use of online games and social skills $(\mathrm{r}=-0.215, \mathrm{p}<0.01)$. This means that the greater use of smaller online games are social skills.
\end{abstract}

Keywords: social skills, chat, online gaming, communication.

\footnotetext{
${ }^{1}$ Estudiante del $4^{\circ}$ año de Psicología

${ }^{2}$ Estudiante del $4^{\circ}$ año de Psicología

${ }^{3}$ Docente de la Facultad de Ciencias de la Salud de la Universidad Peruana Unión
} 


\section{INTRODUCCIÓN}

Sin lugar a dudas, Internet es una de las últimas tecnologías que rápidamente se está desarrollando para su introducción en los hogares. Las previsiones apuntan a su integración como un electrodoméstico más, con unas capacidades y servicios que evolucionarán rápidamente, que se ve reflejado en la última encuesta realizada por Miniwatts Marketing Group, en la cual muestra que solo en América Latina y el Caribe hay 175.8 millones de usuarios de internet y en el Perú hay alrededor de 8.084'900 millones de personas (Éxito Exportador, 2009). Por este motivo, cada vez adquieren más importancia las respuestas de los usuarios frente a la implantación de estas nuevas tecnologías. En este sentido, conviene detenerse un momento en valorar algunos efectos que está produciendo la irrupción de Internet en las conductas de los usuarios.

En efecto, la rápida expansión de la red y su uso cada vez masivo, implica también la necesidad de dedicar un espacio de tiempo diario a utilizar Internet, que puede conllevar en algunos casos un reajuste del tiempo dedicado a otras actividades cotidianas.

Los resultados obtenidos por la última encuesta realizada por la Asociación para la Investigación de los Medios de Comunicación (AIMC, 2001) apuntan ya en esa dirección. Según este informe, un $25 \%$ de las 43'942 personas encuestadas reconoce que ha disminuido el tiempo dedicado a dormir, un $13.8 \%$ ha reducido el tiempo dedicado al estudio, un $10.1 \%$ el tiempo dedicado a salir con los amigos y un $6.1 \%$ dice que el uso de Internet ha disminuido el tiempo laboral.

Y dentro del campo del Internet uno de los servicios más utilizados es el chat (IRC) teniendo un 33\% de uso de los usuarios (AIMC, 2005). Asimismo, en la investigación realizada por Balderas (2006) acerca de las salas de chat como medio de Comunicación Interpersonal el $60 \%$ de los encuestados expresaron que un aspecto negativo del chat es la pérdida de tiempo que conlleva estar en él, siendo los jóvenes de entre 15 a 26 años los usuarios más numerosos. Además en los resultados de la investigación acerca de los problemas conductuales relacionados con el Internet el $4.9 \%$ de la población manifestó tener problemas en las relaciones sociales y familiares, un mayor deseo de estar conectados y sentimientos de culpabilidad, disminuyendo el nivel de habilidades sociales que estas personas puedan tener.

Por su parte, Roberts, Smith y Pollack (1996), indican que posiblemente algunos servicios que ofrece Internet, sobre todo los de mensajería instantánea como chat, propiciarían un uso más intenso de la red, de carácter episódico. Esta circunstancia podría afectar a los sujetos que disponen por primera vez de conexión a Internet, sobre todo a los que vienen con una predisposición disminuida de capacidades para socializar con su entorno social, que tienen un uso marcadamente repetitivo.

Asimismo, otro de los servicios usados en Internet son los juegos en red, como lo demuestra los resultados obtenidos por la última encuesta realizada por la Asociación para la Investigación de los Medios de Comunicación (AIMC, 2010) donde el 12\% de las personas (4'732.320 millones) son asiduos utilizadores de los juegos en red abundando juegos como Conter Stricke, Call of Duty entre otros. Esto demanda tiempo, que los jóvenes generalmente "roban" del tiempo dedicado a los estudios y lo cual constituye un problema grave, ya que tiene sus repercusiones en su rendimiento académico, aparición de conductas disfuncionales ya que la mayoría de los juegos incluyen escenas de muerte, masacre en masa, asesinatos y escenas violentas con alto contenido de sangre. Lo cual dificulta la adaptación e interacción sana a su entorno social donde se desenvuelven. Esto se puede observar con un simple visita a algunas cabinas de Internet, donde se podrá observar y escuchar bombas que revientan, disparos, palabras soeces e insultos entre los miembros que conforman el juego lo cual crea hábitos y conductas no apropiadas en jóvenes universitarios que están comenzando una carrera que requiere dedicación y esfuerzo como es la carrera de Ingeniería de Sistemas y Psicología. Este problema se puede agravar aún más cuando es utilizado por los estudiantes como un medio para evadir los problemas y responsabilidades que demanda el estudio de una carrera. Lo cual puede terminar en una adicción y dificultar la manera cómo se van a relacionar con las personas como sus padres, amigos, novios, etc. Entonces, todo ello influye de manera negativa en las habilidades sociales de los jóvenes, los cuales adoptan actitudes negativas hacia los demás, como falta de asertividad, falta de empatía y comprensión de los demás.

A continuación se describen y definen conceptualmente las variables que configuran nuestro problema de investigación:

\section{Habilidades sociales}

Una definición que reúne aspectos cognitivos, conductuales y sociales es la entregada por Ladd y Mize que se refiere a las Habilidades Sociales como "la habilidad para organizar cogniciones y conductas en un curso integrado de acción orientada por metas interpersonales y sociales de un modo culturalmente aceptada" (Arón 1993). 
Rinn y Markle afirman que las Habilidades Sociales "son un repertorio de comportamientos verbales y no verbales a través de los cuales los niños influyen en las respuestas de otros individuos en el contexto interpersonal" (Michelson 1987).

Combs y Slaby las definen como "la habilidad para interactuar con otros en un contexto dado de un modo específico, socialmente aceptable y valorado y que sea mutuamente beneficioso o primariamente beneficioso para los otros" (Arón 1993).

Libet y Lewinson dicen que es "la capacidad para comportarse de una forma que uno sea castigado o ignorado por los demás" (Michelson 1987).

Michelson, Sugai, Wood y Kazdin entregan la siguiente definición operacional:

- Las Habilidades Sociales se adquieren principalmente a través del aprendizaje.

- Las Habilidades Sociales incluyen comportamientos verbales y no verbales, específicos y discretos.

- Las Habilidades Sociales suponen iniciativas y respuestas efectivas y apropiadas.

- Las Habilidades Sociales acrecientan el reforzamiento social.

- Las Habilidades Sociales son recíprocas por naturaleza y suponen una correspondencia efectiva y apropiada.

- La práctica de las Habilidades Sociales está influida por las características del medio.

- Los déficit y excesos de la conducta social pueden ser especificados y objetivados a fin de intervenir" (Michelson 1987).

Los autores Fernández y Carrobles definen las Habilidades Sociales como la "capacidad que el individuo posee de percibir, entender, descifrar y responder a los estímulos sociales en general, especialmente aquellos que provienen del comportamiento de los demás" (Hidalgo1991).

Argyle dice que son "los procesos de selectividad de la información que cada persona realiza en la interacción social y la posterior interpretación que hace de dicha información” (Hidalgo 1991).

Finalmente, Riso las define como "aquella conducta que permite a la persona expresar adecuadamente y combinando los componentes verbales y no verbales de la manera más efectiva posible; oposición y afecto de acuerdo a sus intereses y objetivos, respetando el derecho de los otros e intentando alcanzar la meta propuesta (Hidalgo 1991).

\section{Chat (IRC)}

Chat es una palabra en inglés cuya traducción significa conversar que usualmente se refiere a una comunicación escrita realizada de manera instantánea a través de Internet entre dos o más personas, desde y hasta cualquier parte del mundo. El Chat fue evolucionando y pasó desde los precarios BBS (Bulletin Board System, una de las formas más primitivas de establecer conexiones entre computadoras).

Son muchas las acepciones de la palabra chat, y por lo general agrupa a todos los protocolos que cumplen la función de comunicar a dos o más personas, dentro de éstos los clientes de chat (como, por ejemplo, X-Chat, ChatZilla).

El chat (IRC) (Internet Relay Chat) es un protocolo de comunicación en tiempo real basado en texto, que permite debates en grupo o entre dos personas y que está clasificado dentro de los servicios de comunicación en tiempo real.

Se diferencia de la mensajería instantánea en que los usuarios no deben acceder a establecer la comunicación de antemano, de tal forma que todos los usuarios que se encuentran en un canal pueden comunicarse entre sí, aunque no hayan tenido ningún contacto anterior.

Las conversaciones se desarrollan en los llamados canales de IRC.

\section{Juegos en red}

Son computadoras conectadas a la red, que pueden compartir el mismo juego desde lugares lejanos, mientras tengan conexión a la red interna de juegos. El computador es una de las mejoras plataformas para los juegos en línea. Con ellos se puede compartir entre varios desde distintos lugares. Entre los jugadores se comunican con un sistema de chat. Para acceder a él hay que tener internet. De hecho, ya se habla de verdaderas relaciones virtuales, las que a diferencia de las reales, tienen como requisito ser llevadas exclusivamente por el computador. Es así como grupos de amigos que nunca se han visto las caras pueden compartir muchas horas disfrutando de alguno de los juegos en línea. Tanto es así que hay grupos virtuales que acostumbran a acceder a los mismos juegos en línea. Con el tiempo se empiezan a conocer y entablan fuertes lazos de relación, pero siempre en el plano virtual, sin nunca verse las caras. Lo curioso es que esos jóvenes ven más a sus amigos virtuales que a los reales, básicamente por lo divertido que les parecen los juegos en línea. (Stevenson, 2010).

Si bien fueron los jóvenes los primeros en adherir con esta posibilidad de la jugar, con el tiempo y la diver- 
sidad de juegos en línea, personas de todas las edades han comprendido lo que es e implica esta nueva forma de comunicarse y divertirse. Además de poder conocer amigos y amigas nuevos, ante los que incluso se pueden romper las barreras virtuales, coordinando un encuentro real entre los que se conocieron en la red computacional.

\section{MATERIAL Y MÉTODOS}

La investigación correspondiente es de diseño noexperimental o ex-post-facto, de tipo correlacional. Siendo desde el punto de vista de la naturaleza relacional entre las variables una investigación multivariante.

Las variables de estudio fueron:

- Habilidad Social.

- Frecuencia de uso del Chat.

- Frecuencia de uso de Juegos en Red.

La población lo constituyeron 164 personas que se encontraban cursando primero y segundo años de la carrera de Ingeniería de Sistemas y Psicología de la Universidad Peruana Unión. La mencionada población incluyó a adolescentes y jóvenes de ambos sexos, con edades entre los 15 y 30 años cumplidos al momento de la evaluación.

Para el presente estudio se estimó una muestra no probabilística de muestreo por conveniencia.

\section{Se utilizó tres instrumentos:}

Escala de Habilidades Sociales (EHS). Fue construida por Elena Gismero Gonzales y fue validado por César Ruiz Alva (2007) en el curso de actualización de Pruebas Psicológicas. Este instrumento tiene como objetivo principal evaluar y medir las habilidades sociales y la capacidad de aserción en distintos contextos. Está constituido por 33 items.

Encuesta para medir la frecuencia de uso del chat: Fue construida especialmente para esta investigación en particular, cuyo objetivo es medir la frecuencia de uso del chat en los jóvenes universitarios de 15 a 25 años. Consta de 9 ítems los cuales están codificados en una escala tipo Likert de cuatro puntos (1: nunca; 2: a veces; 3 : muy a menudo; 4: siempre).

Encuesta para medir la frecuencia de uso de los juegos en red: fue construida especialmente para esta investigación, cuyo objetivo es medir la frecuencia de uso de los juegos en red en los jóvenes universitarios de 15 a 25 años. Consta de 14 ítems los cuales están codificados en respuestas dicotómicas.

Primero se seleccionó los instrumentos adecuados para medir las tres variables de la investigación. Los instrumentos fueron aplicados de manera colectiva en 4 sesiones a los estudiantes universitarios seleccionados previamente. Antes a la aplicación de los instrumentos los encuestadores leyeron las instrucciones en voz alta para contestar las preguntas de los cuestionarios. Para contrarrestar el falseamiento de respuestas se garantizó el anonimato para los encuestados.

\section{RESULTADOS}

Tabla 1

Análisis de correlación entre habilidades sociales, juegos en red y chat en universitarios

\begin{tabular}{lcc} 
& Habilidades Sociales & \\
& $\mathrm{r}$ & $\mathrm{p}$ \\
\hline Chat & $-0,419(* *)$ & 0,000 \\
Juegos en Red & $-0,215(* *)$ & 0,006 \\
\hline
\end{tabular}

** La correlación es significativa al nivel 0,01 (bilateral).

Como se puede apreciar en la tabla 1, el coeficiente de correlación producto momento de Pearson nos indica que existe una relación negativa y altamente significativa entre el uso del chat y las habilidades sociales $(\mathrm{r}=-0.419$, $\mathrm{p}<0.00$ ). Esto quiere decir que cuanto menor es el nivel de uso del chat mayor es el nivel de habilidades sociales. Asimismo, existe una relación negativa y altamente significativa entre el uso de los juegos en red y las habilidades sociales $(\mathrm{r}=-0.215, \mathrm{p}<0.0006)$. Esto quiere decir que cuanto mayor es la frecuencia de uso de los juegos en red menor es el nivel de habilidades sociales. 
Tabla 2

Análisis de correlación entre habilidades sociales, juegos en red y chat en universitarios según carrera profesional

\begin{tabular}{llcc}
\hline Carrera a la que pertenece & & \multicolumn{2}{c}{ Habilidades Sociales } \\
& &,$- 407(* *)$ & $\mathrm{p}$ \\
\hline Ingeniería de Sistemas & Chat &,$- 286\left(^{*}\right)$ &, 000 \\
& Juegos En Red &,$- 292(* *)$ &, 010 \\
\hline Psicología & Chat &,- 003 &, 007 \\
& Juegos En Red &, 981 \\
\hline
\end{tabular}

** La correlación es significativa al nivel 0,01 (bilateral).

* La correlación es significante al nivel 0,05 (bilateral).

En la tabla 2 se aprecia que el coeficiente de correlación (" $r$ ") de -0.407 para las variables chat-habilidades sociales es altamente significativo $(\mathrm{p}<0.01)$; por tanto, se rechaza la hipótesis nula, lo cual señala que existe relación entre el chat y las habilidades sociales en los alumnos de Ingeniería de Sistemas. Asimismo, el coeficiente de correlación (" $r$ ") de $\quad-0.286$ para las variables juegos en red-habilidades sociales es altamente significativo ( $<<0.01$ ); por tanto, se rechaza la hipótesis nula, lo cual señala que existe relación entre los juegos en red y las habilidades sociales en los alumnos de Ingeniería de Sistemas.

Respecto a los alumnos de Psicología para variable chat-habilidades sociales, se puede observar que el coeficiente de correlación obtenido de -0.292 es altamente significativo $(\mathrm{p}<0.01)$; por tanto, para este grupo rechazamos la hipótesis nula. Asimismo, para la variable juegos en red-habilidades sociales se observa que el coeficiente de correlación obtenido de -0.003 no es significativo ( $p>0.01)$; por tanto, para este grupo se acepta la hipótesis nula. Lo cual señala que no existe relación entre los juegos en red y las habilidades sociales en los alumnos de Psicología.

Tabla 3

Análisis comparativo del nivel de habilidades sociales según carrera profesional

\begin{tabular}{lccccccc}
\hline Variable & Carrera & $\mathrm{n}$ & Media & DS & $\mathrm{t}$ & $\mathrm{gl}$ & $\mathrm{p}$ \\
\hline HHSS & Ingeniería de Sistemas & 80 & 92,84 & 13,273 & $-2,677$ & 162 &, 008 \\
& Psicología & 84 & 98,30 & 12,844 & & & \\
\hline
\end{tabular}

La tabla 3 permite apreciar que existen diferencias significativas entre las habilidades sociales que presentan los alumnos de Ingeniería de Sistemas con respecto de los alumnos de Psicología $(\mathrm{t}=-2.677, \mathrm{gl}=162, \mathrm{p}<0.08)$. Los valores de la media indican que los alumnos de Psicología presentan mayores niveles de habilidades sociales, así como la desviación estándar muestra que es un grupo poco homogéneo.

Tabla 4

Análisis comparativo del uso de chat según carrera profesional

\begin{tabular}{llccccc}
\hline Variable & Carrera & $\mathrm{n}$ & Rango promedio & Suma de rangos & $\mathrm{U}$ & $\mathrm{p}$ \\
\hline Chat & Ingeniería de Sistemas & 80 & 106,56 & 8524,50 & 1435,500 &, 000 \\
& Psicología & 84 & 59,59 & 5005,50 & & \\
\hline
\end{tabular}

La prueba de contraste U de Mann-Whitney, utilizado para comparar los grupos de estudio permite observar en la tabla 4 que existe diferencias altamente significativas $(U=1435,500 ; p<0.01)$ con respecto a los niveles de uso del chat. Los valores de la media indican que son los alumnos de Ingeniería de Sistemas $(\mathrm{X}=106.56)$ quienes presentan mayor nivel de uso del chat en contraste los alumnos de Psicología $(X=59.59)$. Por tanto, se rechaza la hipótesis nula y se acepta la hipótesis alterna. 
Tabla 5

Análisis comparativo de juegos en red según carrera profesional

\begin{tabular}{clc|c|c|c|c}
\hline Variable & Carrera & $\mathrm{n}$ & Rango promedio & Suma de rangos & $\mathrm{U}$ & $\mathrm{p}$ \\
\hline \multirow{2}{*}{$\begin{array}{c}\text { Juegos } \\
\text { en Red }\end{array}$} & Ingeniería de Sistemas & 80 & 104,94 & 8395,50 & 1564,500 &, 000 \\
& Psicología & 84 & 61,13 & 5134,50 & & \\
\hline
\end{tabular}

En la tabla 5 según la prueba de contraste U de Mann-Whitney, se observa que existe diferencias altamente significativas $(U=1564.500 ; p<0.01)$ con respecto a los niveles de uso del juegos en red. Los valores de la media indican que son los alumnos de Ingeniería de Sistemas $(X=104,94)$ quienes presentan mayor nivel de uso del chat en contraste los alumnos de Psicología $(\mathrm{X}=61,13)$. Por tanto, se rechaza la hipótesis nula y se acepta la hipótesis alterna.

Tabla 6

Comparación de medias de nivel de habilidades sociales, según la frecuencia de uso del chat.

\begin{tabular}{lcccccc}
\hline Niveles de uso & $\mathrm{n}$ & Media & DT & F & $\mathrm{gl}$ & $\mathrm{p}$ \\
\hline Bajo & 55 & 101,75 & 11,765 & 15,671 & 2 &, 000 \\
Promedio & 77 & 95,03 & 12,672 & & 161 & \\
Alto & 32 & 86,59 & 11,986 & & & \\
\hline
\end{tabular}

Los resultados del análisis de varianza indican en la tabla 6, que existe diferencias altamente significativas en el nivel de habilidades sociales con la frecuencia de uso del chat $(\mathrm{F}=15,671, \mathrm{gl}=2$ y $161, \mathrm{p}<0.01)$. Los valores de la media indican que son los que menos utilizan el chat $(\mathrm{X}=101,75)$ quienes presentan mayor nivel de autoestima en comparación a los que hacen un uso alto del chat $(X=30,47)$.

\section{DISCUSIÓN}

Ante la creciente preocupación para conocer los efectos de Internet y las herramientas (chat y juegos en red) que ofrece, han surgido diferentes estudios en otros países dirigidos a analizar las posibles consecuencias negativas en el bienestar psicológico, las habilidades sociales, entre otros. (Martínez, Cifre y Salanova, 2002).

Muchos son los profesionales dedicados a estudiar este fenómeno que coinciden en afirmar que, sin lugar a dudas, Internet está transformando la vida económica y social, aunque se muestran en desacuerdo acerca de la naturaleza de los cambios que produce y de su dirección beneficiosa o perjudicial (Anderson, Law y Mitchel, 1995; King y Kraemer, 1995). Siendo un hallazgo importante del estudio su relación con el nivel de uso del chat en los estudiantes de pregrado. Encontramos que los estudiantes de las edades de 15 a 19 años se caracterizan por utilizar frecuentemente el chat $(49,5 \%)$.

En otro estudio llevado a cabo con una muestra de 27.2 millones de personas en México se encontró que el $77.3 \%$ de los cibernautas mexicanos tiene menos de 35 años; de modo similar Balderas (2006) en su investigación acerca de "Las Salas de Chat de Internet como medio de Comunicación Interpersonal" reporta los usuarios más numerosos son jóvenes de entre 15 y 25 años. Todos estos datos nos muestran una clara tendencia de que son los jóvenes quienes más uso hacen del chat, y también los primeros que las adoptan. Y este dato es muy importante, ya que a esta edad el ser humano está aún en el proceso de desarrollo y consolidación de sus capacidades y habilidades entre ellas las habilidades sociales. Y el hecho de que estos adolescentes y jóvenes sean los que más uso hagan del chat implica que dediquen horas para su uso dejando de lado otras actividades como salir con amigos, participar de eventos sociales, pasar tiempo con los padres y que en estas actividades los jóvenes desarrollan y consolidan sus habilidades sociales ya que desarrollan y consolidad su capacidad de entender, descifrar y responder a estímulos sociales que no son otra cosa que la actividad comportamental de sus pares.

Pero cuando dedican más tiempo al uso del chat no se ejercitan las habilidades comunicativas porque no hay una comunicación directa con sus pares solo hay un monitor, un teclado y un mouse como mediador de la 
comunicación que en su mayoría es puramente escrita, donde no se da lugar al lenguaje verbal, el cual constituye el casi el $90 \%$ de la comunicación entre dos o más personas.

Así estos jóvenes corren el riesgo de presentar problemas en su interacción social ya que no consolidan la capacidad de detectar y responder al lenguaje verbal y no verbal. Esta situación se agrava más ya que las últimas estadísticas nos muestran que la edad de inicio para el Internet y sus diversas herramientas va descendiendo, así podemos ver niños de 7 u 8 años metidos en una cabina la mayor del tiempo, tiempo que pudieran dedicar a otras actividades como jugar con los amigos, donde se desarrolla la capacidad de autoexpresión, defensa de los propios derechos, expresión de enfado o de conformidad y decir no y cortar interacciones, los cuales son las dimensiones de la habilidad social según Gismero.

Siguiendo con los datos de acerca del uso del chat, juegos en red y el nivel de las habilidades sociales, nuestro estudio comparó algunas de las variables sociodemográficas asociadas. Se encontró mayor prevalencia en los alumnos de Ingeniería de Sistemas, en cuanto al uso del chat (50\%). Entre tanto los estudiantes de Psicología presentaron mayor índice en las habilidades sociales $(97.6 \%)$ al igual que los estudiantes de Ingeniería de Sistemas (91.3\%), las edades de 15 a 19 años presentó un nivel de habilidades sociales (94.5\%) similar al grupo de 20 a 25 (94.5\%) años. Asimismo, los alumnos de Psicología se caracterizan por participar muy poco en los juegos en red (96.4\%). Por otro lado, encontramos mayor prevalecía en estudiantes varones, en cuanto al uso del chat (54.8\%). Los datos se aproximan al estudio de Sánchez, Sánchez y Romero (2000) con 113 estudiantes universitarios donde los varones se caracterizan por utilizar frecuentemente el chat $(36.62 \%)$.

En cuanto a la relación entre el uso del chat, juegos en red y las habilidades sociales se encontró relaciones significativas. Observándose de manera especifica que cuanto mayor es el uso de del chat menores son las habilidades sociales $(r=-0.419 ; \mathrm{p}<0.01)$. Asimismo, nuestros resultados indican que cuanto mayor son las habilidades sociales menor es el uso de los juegos en red, pues como sostienen García Blanco, Vigo, Fernandez y Marco (2002) en su investigación de los problemas conductuales relacionados con el Internet con sus diversas herramientas (chat y juegos en red), nos dice que los problemas más destacados son: los efectos negativos sobre las relaciones sociales o familiares, la reducción de otras actividades cotidianas por estar conectado a internet.

Otro de los hallazgos al relacionar el uso del chat, juegos en red y las habilidades sociales con la facultad a la que pertenecen, fue que los estudiantes de Ingeniería de Sistemas en cuanto al uso del chat presentan correlaciones altamente significativas y negativas $(-0.407$; $\mathrm{p}<0.01)$ con las habilidades sociales, es decir que, cuanto mayor sean las habilidades sociales, habrá menor probabilidad de pasar tiempo en el chat. Asimismo, con los estudiantes de Psicología en cuanto al uso del chat presentan correlaciones altamente significativas y negativas $(-0.292 ; \mathrm{p}<0.01)$ con las habilidades sociales, es decir que, cuanto mayor sean las habilidades sociales, habrá menor probabilidad de pasar tiempo en el chat. Pero en cuanto a los juegos en red no presentan correlaciones significativas $(-0.003 ; \mathrm{p}>0.05)$, con las habilidades sociales.

Esto es de esperarse, debido a que los estudiantes de dicha carrera desde los primeros años llevan cursos y realizan actividades que fortalecen sus habilidades sociales como técnicas terapéuticas, dinámica de grupos, realización de talleres, proyección a la sociedad así como reuniones frecuentes de estudiantes entre las distintas facultades, lo cual fortalecen sus habilidades sociales independientemente de que juegan o no juegos en red, a diferencia de los estudiantes de Ingeniería de Sistemas.

Esto se confirma con los resultados obtenidos cuando se comparan la media de las habilidades sociales en los estudiantes de Psicología e Ingeniería de Sistemas $(\mathrm{t}=-2.677, \mathrm{gl}=162, \mathrm{p}<0.08)$. Los valores de la media indican que los alumnos de Psicología presentan mayores niveles de habilidades sociales. 


\section{REFERENCIAS BIBLIOGRÁFICAS}

1. AIMC Asociación para la Investigación de Medios de Comunicación (2006). Recuperado de http://www.aimc.es.

2. AIMC Asociación para la Investigación de Medios de Comunicación (2005). Recuperado de http://www.aimc.es.

3. AIMC Asociación para la Investigación de Medios de Comunicación (2010). Recuperado de http://www.aimc.es.

4. Éxito Exportador Estadísticas mundiales sobre el Internet (2009). Recuperado de http://www.exitoexportador.com/stats.htm.

5. Hernández, R.; Fernández, C. \& Baptista, P. (1998). Metodología de Investigación. México D.F.: Mc Graw Hill.
6. Michelson, L., Sugai, D.P., Wood, R.P. \& Kazdin, A.E. (1987) Evaluación y tratamiento. Barcelona: Martínez Roca.

7. Rich \& Schoeder (1996). Habilidades Sociales y autocontrol en la adolescencia de Arnold P. Goldtein. Madrid.

8. Roberts, L. D., Smith, L. M., Pollack, C. (1996). A model of social interaction via computer-mediated communication in real-time text-based virtual environments. Paper presented at the annual meeting of the Australian Psychological Society, Sydney, Australia, September.

9. Martinez, Salanova, M. \& Cifred, E. (2001). Time Pressure, Subjective Well-Being and Task Performance among Electronic. Occupational Health Pyscologic: Europe, I-WHO Publications, 58-61 